\title{
Entrainment of the Melatonin Rhythms in Early Postnatal Lambs and Their Mothers
}

\author{
Ruth I. Wood, Lee E. Claypool, ${ }^{1}$ Francis J. P. Ebling, ${ }^{2}$ and Douglas L. Foster ${ }^{3}$ \\ Reproductive Sciences Program, Departments of Physiology, Obstetrics \\ and Gynecology, and Biology, The University of Michigan, \\ Ann Arbor, Michigan 48109-0404
}

\begin{abstract}
Although the developing sheep can produce an appropriately timed melatonin rhythm as early as 1 week after birth, it is not known whether the lamb is able to adjust its melatonin rhythm to a change in daylength. The ability of the young lamb to entrain its pattern of melatonin secretion to a new photoperiod was determined in the present study. Eight female lambs and their mothers were raised in long days (LD 16:8) beginning 2 weeks postpartum. At 7 weeks of age, the time of lights-off was advanced $8 \mathrm{hr}$, the short-day photoperiod then being LD 8:16; the time of lights-on remained unchanged. Concentrations of melatonin were measured in blood samples collected hourly on days $-1,0,2,4,6$, and 13 relative to the light change. On day 0 , all mothers and daughters had advanced the onset of melatonin secretion by at least $1 \mathrm{hr}$, and by day 13,12 of 16 had completely entrained to the new photoperiod. The rate of entrainment among individuals varied; the mean rate for lambs and mothers did not differ. This study provides evidence that the melatonin-rhythm-generating system matures shortly after birth.
\end{abstract}

To begin reproductive cycles at an appropriate time of year, the young female sheep uses its pattern of pineal melatonin secretion to transduce information about length of day to the neuroendocrine system governing reproduction. The lamb monitors the changing seasons via the duration of melatonin secretion (Foster et al., 1986; Yellon and Foster, 1986) and employs the transition from the long days of summer to the shorter days of autumn to time puberty. In artificial lighting conditions, when young females raised on short days are exposed to only 5 weeks of long days from 17 to 22 weeks of age, they undergo puberty at the normal time several weeks later (about 30-35 weeks of age; Foster, 1983; Yellon and Foster, 1985). However, the same long-day to short-day transition during the first 10 weeks of life does not induce normal reproductive cycles (Yellon and Foster, 1985). Rather, puberty is delayed and occurs some time after 1 year of age, much the same as if the lamb had experienced no change in photoperiod.

1. Present address: Wisconsin Regional Primate Research Center, 1223 Capitol Court, Madison, Wisconsin 53715.

2. Present address: Department of Anatomy, University of Cambridge, Cambridge, UK.

3. To whom all reprint requests and correspondence should be addressed, at Room $1101 \mathrm{SW}, 300$ North Ingalls Building, The University of Michigan, Ann Arbor, Michigan 48109-0404. 
The ineffectiveness of this photoperiod treatment during the neonatal period raises the possibilities that at a young age the pineal cannot respond appropriately to changes in photoperiod, or that the reproductive system is not capable of responding to input from the pineal gland. With regard to the first possibility, the lamb can readily produce an appropriately timed melatonin rhythm by $1-6$ weeks of age-a rhythm in which the duration of increased melatonin secretion corresponds to the hours of darkness (Claypool et al., 1989). Whether the very young lamb is able to adjust its melatonin rhythm to a change in daylength is not known. In the present study, the rate of entrainment of melatonin to a novel photoperiod in lambs during the early postnatal period was compared to that of their mothers, to determine whether developmental differences exist in the dynamic regulation of melatonin secretion.

\section{MATERIALS AND METHODS}

\section{ANIMALS AND MAINTENANCE}

Eight female Suffolk lambs (mean $\pm S E M$ birthdate, April $2 \pm 3$ days; range, March 29-April 6) and their mothers were studied indoors in light-sealed rooms with controlled photoperiod. Alfalfa hay and water were provided ad libitum; lambs' diets were supplemented with a commercial pellet ration (Lamb 20, Kent Feed Inc., Muscatine, IA). Mothers and lambs were group-housed from 1 week postpartum until weaning at 8 weeks of age (day 7 of the experiment; see below). Thereafter, the mothers were moved to a separate room with an identical lighting schedule. Beginning at 1 week of age, lambs were weighed weekly to monitor growth (Fig. 1).

\section{PHOTOPERIOD TREATMENTS AND SAMPLE COLLECTION (Fig. I)}

Lighting in photoperiod control rooms was provided by fluorescent tubes emitting approximately 350 lux. A dim red light of $<5$ lux remained on continuously to aid nighttime blood sample collection. Temperature in the rooms was not controlled and varied with the outdoor temperature. Circulating melatonin concentrations were monitored in both mothers and their lambs as they entrained to a new daylength. The photoperiod was changed when lambs averaged 7 weeks of age, on May 20 (Fig. 1). At this time, lambs and their mothers had been maintained for 6 weeks on long days (LD 16:8; lights-on at $0600 \mathrm{hr}$ EST). On day 0 of the experiment, the lights were changed to short days (LD 8:16, lights-on at $0600 \mathrm{hr}$ EST). The $8 \mathrm{hr}$ of darkness were added to advance the time of lights-off, as opposed to a symmetrical 4-hr increase in darkness at both light-dark (LD) transitions. In addition to management considerations, this unidirectional shift was chosen to simplify analysis of entrainment, in that the melatonin rhythms should advance only in a single direction.

Adult females require a week to entrain their melatonin rhythm after an 8-hr light shift from long to short days (Bittman et al., 1983; Malpaux et al., 1987); equivalent information was not available at the time of the study for the interval needed to re-entrain the rhythm of young lambs. Thus, blood samples $(3 \mathrm{ml})$ were 


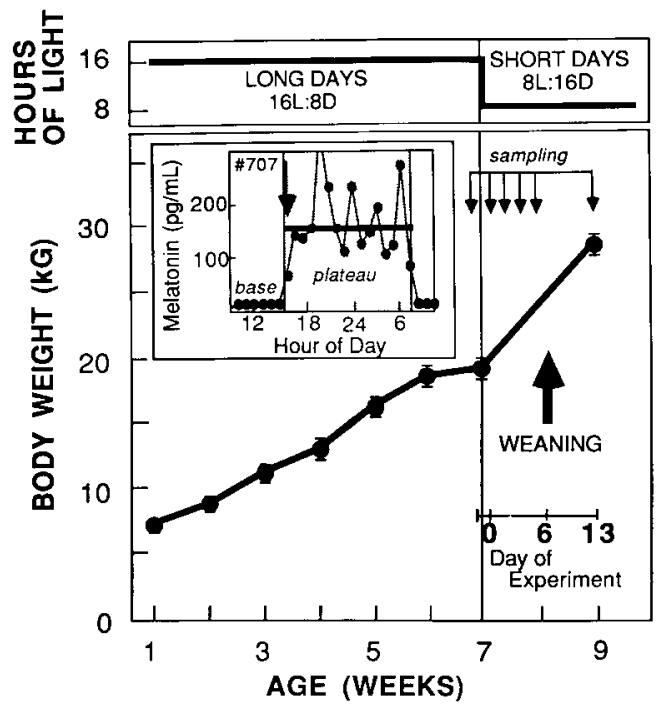

FIGURE 1. Age and body weight (mean $\pm S E M$ ) in lambs during the experimental period. Left panel indicates duration of long-day treatment; right panel indicates short-day photoperiod. Small arrows indicate days of blood sampling for melatonin; larger arrow is the time of weaning. Inset: Serum melatonin profile of a representative lamb to illustrate the onset of melatonin secretion (arrow) as defined in "Materials and Methods"; the shaded area illustrates the hours of darkness. The horizontal bar represents the mean nocturnal melatonin concentration.

obtained hourly for $24 \mathrm{hr}$ by jugular venipuncture on various days from all animals over a 2-week period after the photoperiod shift, in the event that the lambs entrained at a much slower rate than the mothers. Melatonin patterns were monitored in both lambs and their mothers on the day of the light shift (day 0), the previous day (day -1), and on days 2, 4, 6, and 13 after the light shift (Fig. 1). Circadian melatonin patterns were determined on days $-1,0,4$, and 13 . To minimize blood loss from the lambs, hemicircadian patterns of melatonin (hourly blood samples from 1200 to 2300 $\mathrm{hr}, \mathrm{EST}$, spanning the new dusk) were obtained on days 2 and 6. Within $48 \mathrm{hr}$ after collection, samples were centrifuged, and sera were frozen until assayed for melatonin.

\section{MELATONIN ASSAY}

Melatonin was measured by modification (Foster et al., 1989a) of an assay described previously (English et al., 1986). The mean sensitivity, defined as two standard deviations from the buffer control, was $9 \pm 4 \mathrm{pg} / \mathrm{ml}$ (44 assays). Intra-assay coefficient of variation $(\mathrm{CV})$, determined from three pools of standard sera measured four times in each assay, averaged $9 \pm 3 \%$; interassay CV averaged $20 \pm 4 \%$.

\section{DATA ANALYSIS}

To determine the rate of melatonin entrainment, it was necessary to define the initial nocturnal increase in circulating melatonin. Each circadian pattern was analyzed by 
a cluster analysis algorithm (Brown et al., 1988). This method of analysis divides the data points into baseline and plateau clusters. The first data point included in the plateau marks the onset of melatonin secretion (see Fig. 1 for example of nocturnal melatonin onset). The mean onset of melatonin secretion for lambs and mothers was compared using a two-way analysis of variance (ANOVA). In addition, an exponential curve was fitted to these data with the aid of Cricket Graph version 1.2 (Cricket Software, Malvern, PA); this curve predicts the time required to entrain completely to the new hours of darkness.

\section{RESULTS}

Figure 2 illustrates mean concentrations of serum melatonin for lambs and mothers on long days (day -1 ), on the day of the shift to short days (day 0 ), and on days 4 and 13, the days for which full 24-hr patterns of melatonin were available. Figure 3 presents nightly levels of melatonin from two lambs that span the range of rates of entrainment observed in this study. Before the light shift, during exposure to long days, melatonin rhythms of both lambs and their mothers were fully entrained to the LD cycle (day - 1, Fig. 2). Although lights-off was at $1500 \mathrm{hr}$, the earliest that a

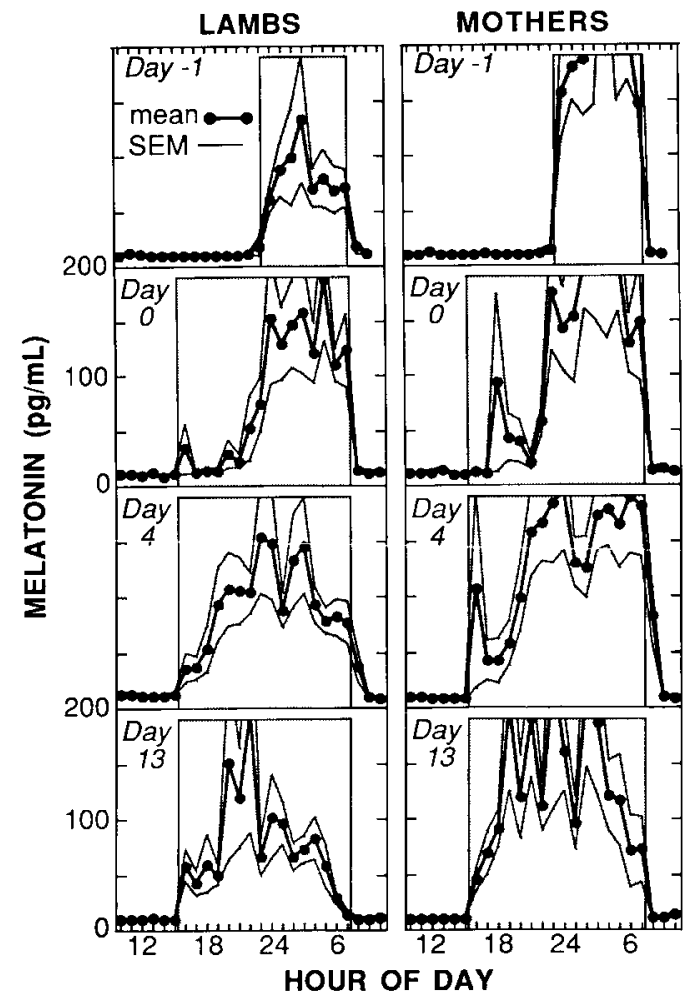

FIGURE 2. Patterns of circulating melatonin (mean $\pm S E M$ ) during each 24-hr collection period for eight lambs (left) and their mothers (right). Shaded areas depict periods of darkness. On day 0 , the photoperiod was shifted to short days. 
melatonin rise would be detected was at $1600 \mathrm{hr}$ in animals completely entrained to the new photoperiod. In our protocol for blood sampling, the sample at $1500 \mathrm{hr}$ was obtained a few minutes before darkness, and the next sample at $1600 \mathrm{hr}$ was the first collected after lights-off. Likewise, because of the method of sample collection, the melatonin rise on day -1 was first apparent at $2400 \mathrm{hr}$ in most animals, despite the onset of darkness at $2300 \mathrm{hr}$. Beginning on day $\mathbf{0}$, both lambs and mothers gradually advanced the onset of melatonin secretion until the rise of melatonin corresponded to the onset of darkness. By day 13, mean serum concentrations of melatonin remained elevated for the duration of the night. It should be noted that weaning on day 7 of the experiment had no discernible effect on the day 13 melatonin pattern.

There was considerable individual variation in rate of entrainment of melatonin secretion, as shown in Figure 3. In one mother-daughter pair (\#724), entrainment was nearly complete on the first day after the light shift (day 0). A more slowly entraining pair (\#701), showed little, if any, evidence of entrainment to the new photoperiod until day 13 , the last day of the study. The rapidly fluctuating concen-

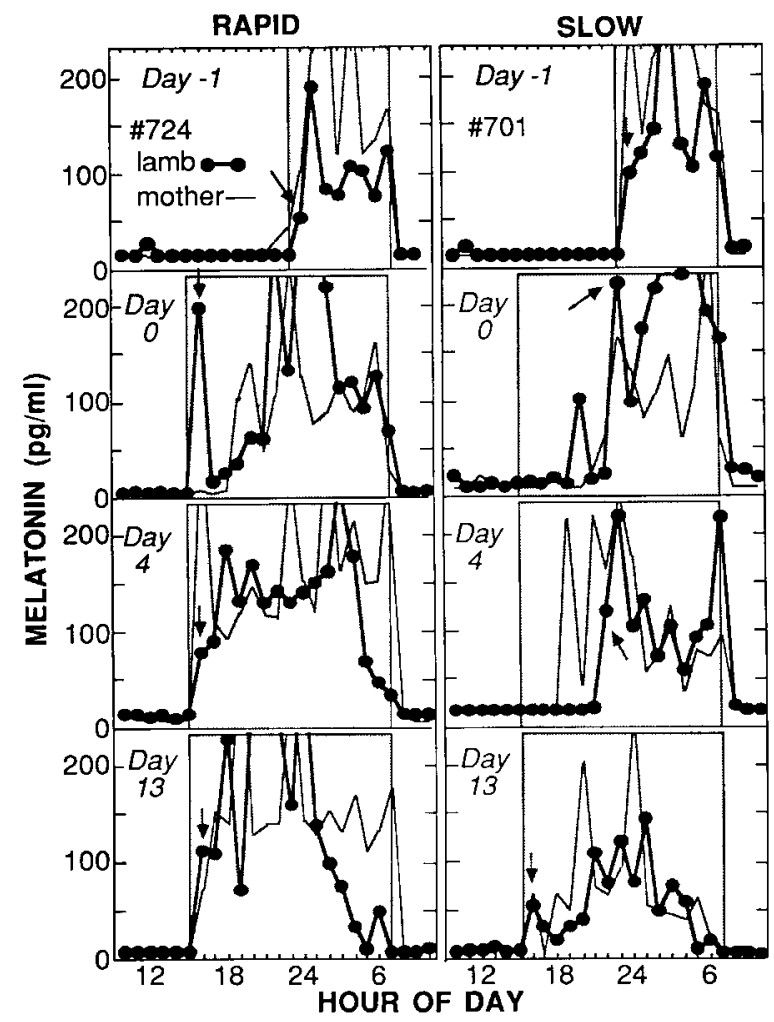

FIGURE 3. Patterns of circulating melatonin for two representative pairs of lambs (bold lines) and their mothers (light lines). Lamb and mother \#724 (left) were the most rapidly entraining pair. Pair \#701 illustrates relatively slow entrainment to the light shift. Arrows indicate the onset of melatonin secretion in the lamb as determined by the clustering analysis algorithm. Shaded areas depict periods of darkness. On day 0 , the photoperiod was shifted from long days to short days. 
trations of melatonin produced considerable variation in amplitude of the nocturnal rises both within and between individuals over time. Although the amplitude of nocturnal melatonin secretion was greater in the mothers than in the lambs, it appears that the reduced amplitude of the lambs is adequate, because supplementation of the nocturnal melatonin rise in the neonatal lamb to adult levels does not influence the subsequent timing of puberty (Foster et al., 1989b).

Figure 4 presents a comparison of mean rates of entrainment for lambs and their mothers. As determined by two-way ANOVA, the rates of entrainment of these two groups did not differ. The entrainment of individual lambs and their mothers, as determined from the nightly onset of melatonin secretion, is depicted in Figure 5. Large individual variation was present, despite the apparent overall similarity between mothers and lambs. Beginning on day 0 , the day the photoperiod was changed, the onset of melatonin secretion progressed forward at least $1 \mathrm{hr}$ in all mothers and seven of eight lambs. By day 13, seven lambs and five of eight mothers had completely entrained to the new photoperiod; only one mother-lamb pair (\#724) had stably entrained before that day (lamb, day 0 ; mother, day 2).

Comparison of the entrainment of the eight pairs of animals revealed no significant correlation between rates of entrainment of individual daughters and mothers $\left(R^{2}=0.10\right)$.

\section{DISCUSSION}

The present study reveals no difference between 7 -week-old lambs and their mothers in the time required for the melatonin rhythm to adjust to a novel photoperiod. Entrainment of both lambs and mothers to an 8 -hr light shift required between 7 and 13 days for most individuals. This agrees with previous reports in adult female sheep,

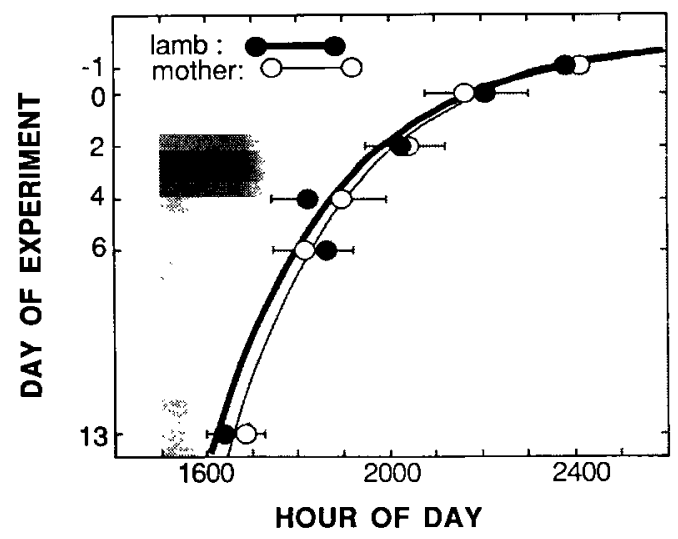

FIGURE 4. Progression of melatonin entrainment in lambs (closed symbols) and mothers (open symbols), plotted as the first hour of elevated melatonin secretion on each night over the course of the experimental period (mean $\pm S E M, n=8$ at each point). The exponential curves for the entrainment of lambs and their mothers are not different. The shaded area indicates the hours of darkness between $1400 \mathrm{hr}$ and $0100 \mathrm{hr}$ on each day of the experiment. 


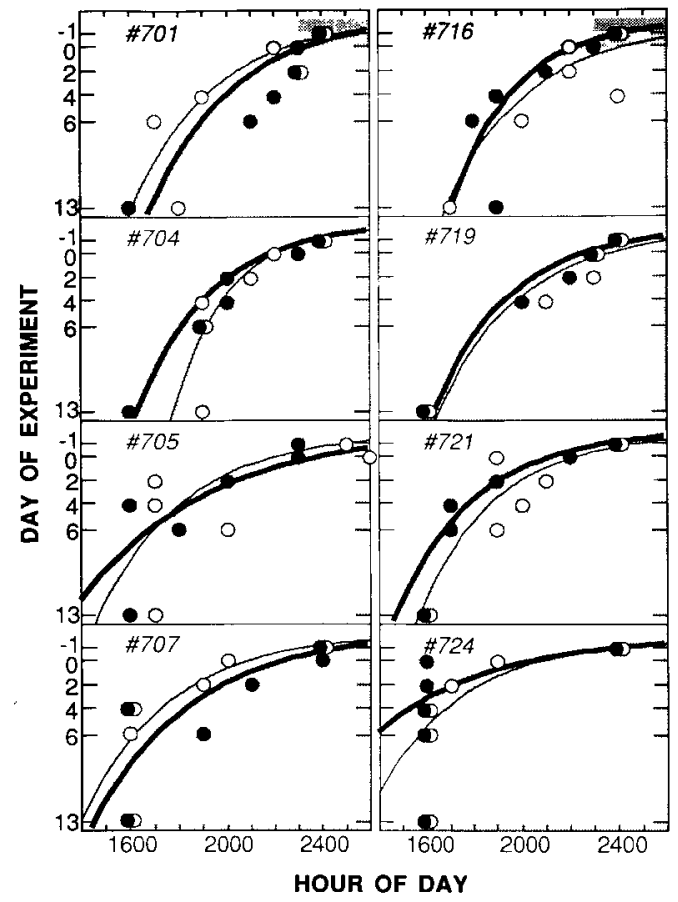

FIGURE 5. Melatonin entrainment of individual lambs (closed symbols) and mothers (open symbols) throughout the experiment. Each individual point represents the first hour of elevated melatonin secretion on each night over the course of the experimental period. The shaded area indicates the hours of darkness between $1400 \mathrm{hr}$ and $0100 \mathrm{hr}$ on each day of the experiment. There was no significant correlation $\left(R^{2}=0.10\right)$ between the time constant of entrainment of a lamb and her mother.

which entrain to an 8-hr light change within 8 days (Malpaux et al., 1987) or 14 days (Bittman et al., 1983).

One explanation for the lack of difference in entrainment rate between mothers and lambs is maternal communication of melatonin to the lamb. Direct passage of melatonin from the ewe to the lamb has been demonstrated to occur before birth by placental transfer (Kennaway et al., 1981; Yellon and Longo, 1987; Zemdegs et al., 1988). After birth the transfer of melatonin from mother to offspring may also be possible, because melatonin is present in milk and can be absorbed from the digestive system, at least in the rat (Reppert and Klein, 1978). Although this is not known for the sheep, there was no perturbation of the melatonin rhythm of the lamb after weaning on day 7 . In this regard, one would expect to see a decrease in the amplitude of melatonin secretion if maternal melatonin augments melatonin concentrations in the lamb. This was not the case. In addition, if the melatonin rhythm in the nursing lamb were of maternal origin, the nocturnal rise of the lamb would be expected to lag behind that of the mother. However, the onset of the nocturnal melatonin rise in a lamb preceded that of her mother by at least $1 \mathrm{hr}$ on 15 of $40(38 \%)$ occasions during the preweaning sampling period (days $-1,0,2,4$, and 6). Finally, in neonatal rats, maternal cues are no longer effective in entraining the activity of $N$-acetyltrans- 
ferase, once the circadian system receives direct retinal innervation by 6 days after birth (Duncan et al., 1986). Such innervation must occur early in the lamb, because in some individuals a competent melatonin rhythm has been identified by 1 week of age (Claypool et al., 1989). From the present results in the lamb, as in the rat, maternal transfer of melatonin is unlikely to play a major role in the generation of a rhythm of melatonin postnatally.

Why is the young lamb unable to respond reproductively to a stimulatory photoperiod cue early in life? When a long-day to short-day photoperiod transition is provided after 20 weeks of age, the melatonin rhythm is already well established (Foster et al., 1986). If the lamb were unable to produce a clear day-night pattern of melatonin earlier, the same photoperiod transition at a younger age would not initiate puberty at the normal time, because the pineal would be unable to transduce the stimulatory photoperiod cue. However, it is now clear that very young lambs are capable of producing a melatonin pattern appropriate to the photoperiod (Claypool et al., 1989; present study). Moreover, the results of this study cannot account for the failure of puberty to occur in response to the shift from long days to short days early in life, because the lamb also attains the ability to entrain rapidly to a new photoperiod early in life.

Finally, it should be noted that photoperiod information is clearly perceived by the young lamb to influence hormone secretion. Serum prolactin concentrations were found to be higher throughout the first few weeks after birth in lambs exposed to long days than in lambs kept on short days; this difference was prevented by denervation of the pineal gland (Ebling et al., 1988). Furthermore, as shown in gonadectomized female lambs, within a few weeks after birth long-day photoperiod has a stimulatory effect on luteinizing hormone pulse frequency in the absence of steroid negative feedback (Ebling, unpublished results).

Perhaps perception of and early responses to photoperiod are unimpaired, as inferred earlier, but other neuroendocrine pathways to the reproductive system indicate that somatic size is inadequate. In this regard, the nutritionally growthrestricted lamb remains hypogonadotropic, and puberty cannot occur (Foster and Olster, 1985), despite the lamb's ability to perceive seasonal photoperiod cues (Foster and Yellon, 1985). It appears that the requirements for growth and for photoperiod must be satisfied concurrently before puberty will occur. According to this hypothesis, lambs do not experience accelerated puberty when exposed to a longday to short-day photoperiod transition early in life, because growth is limiting.

\section{ACKNOWLEDGMENTS}

This research was supported by grants from the National Institutes of Health (Nos. HD-07048, HD-18258, HD-18394, and biomedical support to the Vice President for Research at The University of Michigan); a fellowship from the Regents of The University of Michigan (to Ruth I. Wood); and a Fulbright Travel Award and Fogarty Fellowship (No. TW03766 to Francis J. P. Ebling). We are grateful to Mr. Lee H. Breasbois (Freeland, Michigan) for providing high-quality lambs for experimentation; Mr. Kirk C. Van Natter of the Reproductive Sciences Program Sheep Research Facility for conscientious animal care; Mr. Douglas D. Doop and Ms. Tovaghgol E. Adel for expert technical advice and assistance; Dr. Morton B. Brown for assistance with statistical analyses; Mr. Michael R. Muha for microcomputer support; Ms. Betty C. Lewis for manuscript typing; the Reproductive Sciences Program Standards and Reagents Core 


\section{EARLY MELATONIN ENTRAINMENT}

Facility for standardization of hormone radioimmunoassay reagents; Dr. Josephine Arendt, University of Surrey, for the melatonin antiserum used in radioimmunoassays; and Dr. David C. Bucholtz and Ms. Lesley A. Vannerson for their help in experimental design and blood sample collection.

\section{REFERENCES}

Bittman, E. L., R. J. Dempsey, and F. J. KARSCH (1983) Pineal melatonin secretion drives the reproductive response to daylength in the ewe. Endocrinology 113: 2276-2283.

Brown, M. B., F. J. Karsch, and B. Malpaux (1988) An algorithm to identify changes in hormone patterns. In Proceedings of the XXth Interface on Computer Science and Statistics, pp. 779-784.

Claypool, L. E., R. I. Wood, S. M. Yellon, and D. L. Foster (1989) The ontogeny of melatonin secretion in the lamb. Endocrinology 124: 2135-2143.

DUNCAN, M. J., M. J. Banister, and S. M. RePPERT (1986) Developmental appearance of lightdark entrainment in the rat. Brain Res. 369: 326330.

Ebling, F. J. P., L. E. Claypool, and D. L. FosTER (1988) Neuroendocrine responsiveness to light during the neonatal period in sheep. $\mathrm{J}$. Endocrinol. 119: 211-218.

English, J., A. L. Poulton, J. Arendt, and A. M. Symons (1986) A comparison of the efficiency of melatonin treatments in advancing estrus in ewes. J. Reprod. Fert. 77: 321-327.

Foster, D. L. (1983) Photoperiod and sexual maturation of the female lamb: Early exposure to short days perturbs estradiol feedback inhibition of luteinizing hormone secretion and produces abnormal ovarian cycles. Endocrinology 112: 11-17.

Foster, D. L., F. J. P. Ebling, L. E. Claypool, R. I. Wood, T. E. Adel, and W. Schramm (1989a) Amplitude modulation of the nightly melatonin rise in the neonatal lamb and the subsequent timing of puberty. Biol. Reprod. 40: 920928.

Foster, D. L., F. J. Karsch, D. H. Olster, K. D. RYan, and S. M. Yellon (1986) Determinants of puberty in a seasonal breeder. Rec. Prog. Horm. Res. 42: 330-384.
Foster, D. L., and D. H. Olster (1985) Effect of restricted nutrition on puberty in the lamb: Patterns of tonic luteinizing hormone $(\mathrm{LH})$ secretion and competency of the LH surge system. Endocrinology 116: 375-381.

Foster, D. L., and S. M. Yellon (1985) Photoperiodic time measurement is maintained in undernourished lambs with delayed puberty. J. Reprod. Fert. 75: 203-208.

Foster, D. L., S. M. Yellon, F. J. P. Ebling, and L. E. Claypool (1989b) Are ambient shortday cues necessary for puberty in a short-day breeder? Biol. Reprod. 38: 821-829.

Kennaway, D. J., C. D. Matthews, and R. F. Seamark (1981) Pineal function in pregnancy: Studies in sheep and man. In Pineal Function, C. D. Matthews and R. F. Seamark, eds., pp. 123-136. Elsevier/North-Holland, New York.

MalpauX, B., J. E. Robinson, M. B. Brown, and F. J. KARSCH (1987) Reproductive refractoriness of the ewe to inductive photoperiod is not caused by inappropriate secretion of melatonin. Biol. Reprod. 36: 1333-1341.

RePPERT, S. M., and D. C. Klein (1978) Transport of maternal $\left[{ }^{3} \mathrm{H}\right]$ melatonin to suckling rats and the fate of $\left[{ }^{3} \mathrm{H}\right]$ melatonin in the neonatal rat. Endocrinology 102: 582-588.

Yellon, S. M., and D. L. Foster (1985) Alternate photoperiods time puberty in the female lamb. Endocrinology 116: 2090-2097.

Yellon, S. M., and D. L. Foster (1986) Melatonin rhythms time photoperiod-induced puberty in the female lamb. Endocrinology 119: 44-49.

YeLLON, S. M., and L. D. Longo (1987) Melatonin rhythms in fetal and maternal circulation during pregnancy in the sheep. Amer. J. Physiol. 252: E799-E802.

ZemDegs, I. Z., I. C. McMillen, D. W. Walker, G. D. Thorburn, and R. NowAK (1988) Diurnal rhythms in plasma melatonin concentrations in the fetal sheep and pregnant ewe during late gestation. Endocrinology 123: 284. 